\title{
Effects of curcumin on the gene expression profile of L-02 cells
}

\author{
MINGJIE ZHOU, CHUNLEI FAN and NAN TIAN \\ College of Life Science, Zhejiang Chinese Medical University, Hangzhou, Zhejiang 310053, P.R. China
}

Received February 24, 2015; Accepted April 3, 2015

DOI: $10.3892 /$ br. 2015.460

\begin{abstract}
Curcumin has been utilized in the treatment and prevention of disease, including cancer and cardiovascular disease, but the molecular mechanism behind such numerous effects remains unclear. To explore the molecular mechanism and action sites of curcumin at the cellular level, human hepatic L-02 cells were used to assess these effects. Microarray technology was employed to detect the gene expression of L-02 cells treated with curcumin. According to the microarray results and the data from the National Center for Biotechnology Information, the present study classified and concluded that these curcumin-sensitive genes were associated with diseases and their signaling pathway. The study supports the evidence for treating cancer and cardiovascular disease, and found certain potential marker genes for conducting systematic research into the effects of curcumin. A total of 80 genes were identified as significantly differentially expressed between samples treated with and without curcumin. Curcumin is capable of developing physiological reactions and functions by regulating the gene expression and affecting its signal transduction pathway. Tumor protein p63, MYC-associated factor $\mathrm{X}$ and certain other genes associated with tumors act on a potential therapeutic target, while apolipoprotein $\mathrm{B}$ receptor and oxysterol-binding protein-like 7, and their signaling pathways may be involved in the cardioprotective effects of curcumin.
\end{abstract}

\section{Introduction}

Curcumin [1,7-bis (4-hydroxy-3-methoxypheny1)-1, 6-heptadiene-3,5-dione, $\mathrm{C}_{21} \mathrm{H}_{20} \mathrm{O}_{6}$ ] is a hydroalcoholic extracted compound of the traditional Chinese medicine, Rhizoma Curcumae Longae, which has several essential biological functions, including anti-inflammatory, antioxidant, anticarcinogenic, hypercholesterolemia and cardioprotective activities (1-3). Animal studies have suggested that curcumin has a wide range of potential therapeutic effects. Aggarwal et al (3) reported that curcumin, which is responsible for the majority

Correspondence to: Professor Chunlei Fan, College of Life Science, Zhejiang Chinese Medical University, 548 Binwen Road, Hangzhou, Zhejiang 310053, P.R. China

E-mail: snow@zjtcm.net

Key words: curcumin, hepatocyte, gene expression, gene microarray of the biological activity of turmeric, has been used historically as a component of Indian Ayurvedic medicine to treat a wide variety of ailments. Curcumin has been used extensively to treat cardiovascular diseases (4) and cancer (2). According to recent studies, curcumin is an active lipid-mediating compound that can significantly decrease the level of serum lipid peroxides, increase high-density lipoprotein cholesterol (HDL-C) and decrease the total serum cholesterol $(5,6)$.

With the development of microarray technology, it is possible to simultaneously detect the expression of millions of target genes (7). In a previous study, microarray technology was used to detect the differential gene expression of animals treated with curcumin (8). However, the effects of curcumin treatment on the profiles of total gene expression in vitro are seldom reported.

Further studies have exhibited more pharmacological roles of curcumin. There is extensive experimental evidence that curcumin is an important factor involved in the initiation and development of numerous diseases by regulating the expression of genes or activating signaling pathways, but only a few of the functional genes regulated by curcumin are assessed and reported. Jain et al (9) reported that the liver was considered as the major organ responsible for the metabolism of curcumin. Accordingly, it is desirable to compare the gene expression profile on hepatocyte cells L-02 treated with and without curcumin in order to identify the molecular mechanism and action sites of curcumin at the cellular level, subsequently gain highly specific and sensitive drug action marker genes of curcumin. The aim of the present study was to provide the gene expression and possible gene signaling pathways affected in L-02 cells by curcumin treatment.

\section{Materials and methods}

Reagents and chemicals. Curcumin was obtained from the Delta Information Centre for Natural Organic Compounds (Xuacheng, Anhui, China). The L-02 cell line was provided by Shanghai Cell Bank, China Academy of Science. Cell culture medium was from Gibco Invitrogen Life Technologies (Carlsbad, CA, USA). TRIzol was purchased from Invitrogen Life Technologies. The two-step reverse transcription-polymerase chain reaction (RT-PCR) kit obtained from Hangzhou Bioer Technology Co., Ltd. (Hangzhou, China); and reagents regarding DNA microarray and standard protocols were provided by KangChen Bio-tech Inc. (Shanghai, China).

Culture of L-02 and total RNA extraction. i) L-02 hepatocyte cells were seeded in two $25-\mathrm{cm}^{2}$ flasks and cultured in 
RPMI-1640 medium with $10 \%$ bovine calf serum in a $5 \% \mathrm{CO}_{2}$ atmosphere at $37^{\circ} \mathrm{C}$. The medium was refreshed every $24 \mathrm{~h}$. When the cell density reached $\sim 80 \%$ confluence, test samples were treated with the medium containing $20 \mu \mathrm{M}$ curcumin and the control samples were treated with normal medium. ii) After $24 \mathrm{~h}$, total RNA was extracted using the TRIzol reagent according to the manufacturer's instructions. TRIzol reagent $(1 \mathrm{ml})$ was added to each flask and homogenized with a homogenizer. iii) Following extraction with chloroform, RNA was precipitated with isopropanol. The resultant pellet was finally re-suspended in RNAase-free water. iv) Following a DNase digestion step, RNA quantification and quality assurance were assessed by NanoDrop ND-1000 (NanoDrop Technologies, Wilmington, DE, USA) at 260/280 nm and RNA integrity and genome DNA contamination were tested by denaturing agarose gel electrophoresis.

Microarray analysis. Microarray chips, associated protocols and equipment for the processing of these chips were from KangChen Bio-tech Inc. Probe synthesis from the total RNA samples, hybridization, detection and scanning were performed according to standard protocols.

i) First and second strand cDNA were synthesized from total RNA using the two-step RT-PCR kit according to the manufacturer's instructions. Following this, aRNA was synthesized according to recommended instructions by KangChen Bio-tech Inc. ii) Following purification of aRNA, the solution was transferred to NucAway Spin Columns and the aRNA was labeled using CyDye according to the manufacturer's instructions. iii) The efficiency of aRNA labeling was assessed by NanoDrop ND-1000. The efficiency of aRNA labeling = concentration of aRNA labeling/total aRNA. iv) The labeled aRNA was hybridized with a highly sensitive Homo sapiens array according to the standard protocol. Following hybridization, the arrays were washed in a GeneChip Fluidics Station 450. v) After washing and drying, hybridization signals derived from CyDye were scanned with a GenePix 4000B and processed with Agilent array. vi) Subsequent further analysis, including data preprocessing and normalization, differential, statistical and cluster analysis, were performed by software carried in GenePix 4000B.

\section{Results}

The total RNA with A260/A280 value >1.5 was accepted for subsequent use in the DNA chip analysis. The $\mathrm{OD}_{260} / \mathrm{OD}_{280}$ of the test sample was 1.87 while that of the control sample was 1.56 , reaching the standard required.

The aRNA labeling with an A260:A280 value between 1.8 and 2.1 and an efficiency of aRNA labeling of $>0.9$ was accepted for subsequent use in the DNA chip analysis. The efficiency of aRNA labeling treated with curcumin was 0.96 and the control sample was 0.93 , while the quantification and quality of aRNA reached the accepted standard of GeneChip analysis.

The test and control samples were examined by GeneChip and each sample was tested in triplicate to reduce technical errors. Altogether, 6 GeneChips were used to examine the gene expression profile of L-02 cells. Genes with 5-fold higher expression compared to the control were regarded as upregulated genes, while genes with 0.33 -fold lower expression were regarded as downregulated genes.

All gene expression is included in Table I. A total of 80 genes was found to be significantly differentially expressed, of which $47(58.8 \%)$ were upregulated and 33 (41.3\%) downregulated; 21 (26.3\%) were associated with cancer, cell cycle and cell differentiation and $4(5 \%)$ were associated with lipid metabolism and immunoreactions. These genes were classified based upon their functions and primary biological processes by referring to the National Center for Biotechnology Information.

\section{Discussion}

Using DNA microarray, the differences in gene expression profiling of human L-02 cells treated with curcumin compared to the control sample were identified. Future studies should clone and analyze these functional differential genes. After comparing the differential expression of the two samples, the data of the present study indicates the same conclusions as certain other research studies regarding the effects of curcumin, such as the study by Kakar and Roy (10) on the antitumor effect of myc and extensive cardioprotective effects against diabetic cardiovascular complications reported by Hong et al (8). Other novel potential marker genes are also revealed by the present study, such as leption and oxysterol-binding protein-like 7.

Curcumin is capable of developing physiological reactions and functions by regulating the expression and affecting the signal transduction pathway.

The KIAA0937 protein is a regulator of the notch signaling pathway involved in cell-cell communications that regulates a broad spectrum of cell-fate determinations (11). Following treatment with curcumin, the expression of the KIAA0937 protein was only $29.66 \%$ of the control sample, which indicates that curcumin reduced the communication between cells, which may be involved in suppressing metastasis.

Binding of platelet-activating factor (PAF) to the PAF receptor stimulates numerous signal transduction pathways, including phospholipase C, D, A2, mitogen-activated protein kinases and the phosphatidylinositol-calcium second messenger system (12). From the data of the DNA chip, curcumin upregulated the expression of platelet-activating factor receptor (PTAFR). With the increased level of PTAFR, PAF participates in these physiological processes more effectively. Following PAF stimulation, PTAFR becomes phosphorylated, internalized and downregulated, and subsequently the downstream pathway is affected, such as platelet aggregation and degranulation, inflammation and anaphylaxis (13).

RAB1A is a member of the Ras superfamily of guanosine triphosphatases (GTPases) and functions through binding GTP and guanosine diphosphate and possessing intrinsic GTPase activity (14). Phosphorylation and downregulation of RAB1A by curcumin are involved in numerous biological activities. Once these signaling pathways are activated, various physiological phenomena are improved and diseases may be cured.

Curcumin also regulated a few of the genes functioning as transcriptional activators or transcriptional repressors. Zinc fingers and homeoboxes 1 is a member of the zinc fingers and homeoboxes gene family, which belongs to nuclear 
Table I. Gene expression profiles of L-02 cells following treatment with curcumin.

GenBank

Gene description
Fold change

Genes associated

with tumor

NM_003722.4

Tumor protein p63 (TP63) plays a role in the regulation of epithelial morphogenesis

25.01

NM_001319.5

Casein kinase 1, $\gamma 2$ (CSNK1G2) may be involved in gene expression, DNA repair and

NM_004134.6 cell cycle procession

14.16 response and maintenance of the mitochondria

NM_002211.2 Integrin, $\beta 1$ (fibronectin receptor, $\beta$ polypeptide, antigen CD29 includes MDF2, MSK12) (ITGB1) involved in cell adhesion and recognition in a variety of processes

NM_203301.3 F-box protein 33 (FBXO33) regulates to cell phase conversion, signal transduction and the other physiological processes

NM_014994.2 Mitogen-activated protein kinase binding protein 1 (MAPKBP1). Involved in JNK signaling pathway

NM_178275.4 Immunoglobulin-like and fibronectin type III domain containing 1 (IGFN1) can be combined with hepatitis B antigen

XM_208200.5 Predicted: Similar to heterogeneous nuclear ribonucleoprotein A1 (helix-destabilizing protein) (single-strand binding protein) (hnRNP core protein A1) (HDP-1)

(topoisomerase-inhibitor suppressed) (LOC284387)

NM_003779.3 UDP-Gal: $\beta$ GlcNAc $\beta 1$,4-galactosyltransferase, polypeptide 3 (B4GALT3) encodes an enzyme that may be mainly involved in the synthesis of the first $\mathrm{N}$-acetyllactosamine unit of poly-N-acetyllactosamine chains

NM_001005909.2 Inositol hexakisphosphate kinase 2 (IP6K2) encodes a protein that belongs to the inositol phosphokinase (IPK) family, affects the growth suppressive and apoptotic activities of interferon- $\beta$ in certain type of ovarian cancer

NM_020350.3 Angiotensin II receptor-associated protein (AGTRAP) may be involved in cell proliferation and angiotensin II-stimulated transcriptional activity

NM_022148.2 Cytokine receptor-like factor 2 (CRLF2) forms a functional complex with TSLP and IL7R, which is capable of stimulating cell proliferation through activation of STAT3 and STAT5. Also activates JAK2

Genes correlated to lipid metabolism

NM_018690.3

NM_024082.2

Apolipoprotein B receptor mediates the rapid high-affinity uptake of chylomicrons, HTG-VLDL and trypsinized VLDL devoid of APOE in vitro in macrophages Proline rich Gla (G-carboxyglutamic acid) 3 (transmembrane) (PRRG3) encodes a protein that plays an important role in inhibiting vascular calcification

NM_001909.4 Dathepsin D (CTSD) encodes a lysosomal aspartyl protease, mutations in this gene are involved in the pathogenesis of several diseases, including breast cancer and possibly Alzheimer's disease

Genes correlated to immunoreaction

NM_005091.1

Peptidoglycan recognition protein 1 (PGLYRP1) is a pattern receptor, which plays a role in natural immune recognition and killing bacteria

NM_001013722.1 Similar to zinc finger protein 469 (LOC441193), may play a role in protection of endothelia cell injury

NM_000952.4 Platelet-activating factor receptor (PTAFR) encodes a seven-transmembrane GPCR for platelet-activating factor, be a chemotactic phospholipid mediator that possesses potent inflammatory, smooth-muscle contractile and hypotensive activity

NM_000230.2 Leptin (LEP) encodes a protein that plays a major role in the regulation of body weight and is involved in the regulation of immune and inflammatory responses

Genes correlated to other physical activities 
Table I. Continued.

GenBank

NM_005108.2
NM_022068.1

NM_004577.3

NM_002854.2

AY062434

NM_007210.3

BC034757

NM_197965.2

NM_004926.2

NM_030907.3

NM_006729.4

Unknown

NM_001427.2

NM_001099652

AL832422

AK093304

XM_378043.2

AK127789

XM_373535.1

XM_051271.8

AK095652

XM_498484.1

BC080569.1

BX538277

NM_178525.3

NM_005452.5

BM701295

NM_181724.2

Genes associated with tumor

NM_002382.3

NM_004393.4

NM_001006657.1
Gene description

Fold change

Xylulokinase homolog (H. influenzae) (XYLB) may play a role in metabolism

17.06

Piezo-type mechanosensitive ion channel component 2 , the protein has a role in rapidly

16.60

adapting mechanically activated (MA) currents in somatosensory neurons

Phosphoserine phosphatase (PSPH), this encoded enzyme is responsible for the third and last step in L-serine formation. Deficiency of this protein is thought to be linked to Williams syndrome

Parvalbumin (PVALB) encodes a protein that is thought to be involved in muscle relaxation

11.53

11.09 synthesis

10.96 (GalNAc-T6) (GALNT6). The encoded protein may be involved in the synthesis of oncofetal fibronectin

Indian hedgehog homolog (Drosophila) regulates proliferation and differentiation of chondrocytes and is essential for bone formation

Homo sapiens solute carrier family 10 member 6 (SLC10A6) transports sulfo

conjugation steroid hormones, as well as taurolithocholic acid-3-sulfate and

sulfoconjugated pyrenes in a sodium-dependent manner

Zinc finger protein 36, C3H type-like 1 (ZFP36L1), the putative nuclear transcription factor most likely functions in regulating the response to growth factors

REM2 and RAB-like small GTPase 1 (RSG1). Potential effector of the planar cell polarity signaling pathway

Diaphanous homolog 2 (Drosophila) (DIAPH2), could be involved in oogenesis.

Involved in the regulation of endosome dynamics

Homo sapiens engrailed homeobox 2 (EN2), mRNA

26.04

Homo sapiens G protein-coupled receptor 137C (GPR137C), mRNA

24.19

Homo sapiens mRNA; cDNA DKFZp762K012 (from clone DKFZp762K012)

20.02

Homo sapiens cDNA FLJ35985 fis, clone TESTI2014248

11.77

Homo sapiens similar to RIKEN cDNA 1700091F14 (LOC402353), mRNA

10.05

Homo sapiens cDNA FLJ45890 fis, clone OCBBF3022576

9.42

Homo sapiens hypothetical LOC387864 (LOC387864), mRNA

8.89

Family with sequence similarity 10, member A6

8.82

Homo sapiens cDNA FLJ38333 fis, clone FCBBF3025674

7.71

7.45

Predicted: Homo sapiens LOC439971 (LOC439971), mRNA

7.32

Homo sapiens cDNA clone IMAGE: 6537414

6.81

Homo sapiens genomic DNA; cDNA DKFZp68601294 (from clone DKFZp68601294)

6.66

5.80

Homo sapiens WD repeat domain 46 (WDR46), transcript variant 1, mRNA

5.58

UI-E-EJ0-ahj-h-16-0-UI 5-, mRNA sequence

Homo sapiens transmembrane protein 119 (TMEM119), mRNA

5.43

MYC-associated factor X (MAX) is a member of the basic helix-loop-helix leucine

$21.95 \%$ (bHLHZ) family of transcription factors. Myc is an oncoprotein implicated in cell proliferation, differentiation and apoptosis

Dystroglycan 1 (dystrophin-associated glycoprotein 1; DAG1). The dystroglycan complex is involved in a number of processes including laminin and basement membrane assembly, sarcolemmal stability and cell survival

WD repeat domain 35 (WDR35). Members of this family are involved in a variety of cellular processes, including cell cycle progression, signal transduction, apoptosis and gene regulation 
Table I. Continued.

\begin{tabular}{|c|c|}
\hline GenBank & Gene description \\
\hline NM_030877.3 & $\begin{array}{l}\text { Catenin, } \beta \text { like } 1 \text { (CTNNBL1). Although the function of this protein has not been } \\
\text { determined, the C-terminal portion of the protein has been shown to possess } \\
\text { apoptosis-inducing activity }\end{array}$ \\
\hline NM_005762.2 & $\begin{array}{l}\text { Tripartite motif containing } 28 \text { (TRIM28). The protein mediates transcriptional control by } \\
\text { interaction with the Kruppel-associated box repression domain found in numerous } \\
\text { transcription factors }\end{array}$ \\
\hline NM_004861.1 & $\begin{array}{l}\text { Galactose-3-O-sulfotransferase } 1 \text { (GAL3ST1). Activity of this sulfotransferase is } \\
\text { enhanced in renal cell carcinoma }\end{array}$ \\
\hline NM_133646.1 & $\begin{array}{l}\text { Sterile } \alpha \text { motif and leucine zipper containing kinase AZK (ZAK) is a member of the } \\
\text { MAPKKK family of signal transduction molecule, activity of this protein plays a role } \\
\text { in cell cycle checkpoint regulation in cells, and has pro-apoptotic activity }\end{array}$ \\
\hline NM_000964.1 & $\begin{array}{l}\text { Retinoic acid receptor, } \alpha \text { (RARA), has been implicated in regulation of development, } \\
\text { differentiation, apoptosis, granulopoeisis and transcription of clock genes }\end{array}$ \\
\hline \multicolumn{2}{|l|}{$\begin{array}{l}\text { Genes correlated } \\
\text { to lipid } \\
\text { metabolism }\end{array}$} \\
\hline NM_145798.2 & $\begin{array}{l}\text { Oxysterol binding protein-like } 7 \text { (OSBPL7). The encoded protein contains an N-terminal } \\
\text { pleckstrin homology domain and a highly conserved C-terminal OSBP-like sterol-binding } \\
\text { domain }\end{array}$ \\
\hline
\end{tabular}

Fold change

$24.80 \%$

$26.20 \%$

$27.14 \%$

$28.25 \%$

$28.79 \%$

Genes associated with other physical activities NM_005746.2

Nicotinamide phosphoribosyltransferase (NAMPT) is thought to be involved in numerous

NM_004687.4 important biological processes, including metabolism, stress response and aging

Myotubularin related protein 4 (MTMR4) may play a role in the development and normal function of the ovaries

NM_144969.2 Zinc finger, DHHC-type containing 15 (ZDHHC15). Mutations in this gene are associated with mental retardatio X-linked type 91 (MRX91)

NM_002399.3

Meis homeobox 2 (MEIS2) are highly conserved transcription regulators

NM_005754.2

GTPase activating protein (SH3 domain) binding protein 1 (G3BP1) is a member of the heterogeneous nuclear RNA-binding proteins and is also an element of the Ras signal transduction pathway. May be a regulated effector of stress granule assembly

NM_145260.2 Odd-skipped related 1 (Drosophila) (OSR1) plays a role in the regulation of embryonic heart and urogenital development

NM_005277.3 Glycoprotein M6A (GPM6A) may be involved in the differentiation and neuronal migration of neurons

NM_007222.4 Zinc fingers and homeoboxes 1 (ZHX1) encodes member 1 of the family of the zinc fingers and homeoboxes gene family

NM_002373.5 Microtubule-associated protein 1A (MAP1A) encodes a protein that belongs to the microtubule-associated protein family that is involved in microtubule assembly Mex-3 homolog C (MEX3C). The protein interacts with mRNA via the KH domains, may be involved in post-transcriptional regulatory mechanisms

NM_054012.2 Argininosuccinate synthase 1 (ASS1). The protein catalyzes the penultimate step of the arginine biosynthetic pathway

NM_004161.4 Member RAS oncogene family (RAB1A) encodes a member of the Ras superfamily of GTPases

NM_152717.1 Membrane-spanning 4-domains, subfamily A, member 15 (MS4A15) may be involved in signal transduction as a component of a multimeric receptor complex

AB023154

NM_012208.2 mRNA for KIAA0937 protein is a regulator of Notch signaling 
Table I. Continued.

GenBank

Gene description

Fold change

XM_498440.1 Predicted: Homo sapiens hypothetical gene supported by NM_153014 (LOC439926), mRNA

$18.88 \%$

AF130048

Homo sapiens clone FLB3344 PRO0845 mRNA, complete cds

$21.61 \%$

BX537698

Homo sapiens mRNA; cDNA DKFZp686F09166 (from clone DKFZp686F09166)

$22.96 \%$

AK092623

Homo sapiens cDNA FLJ35304 fis, clone PROST2009731

$24.47 \%$

NM_207354.2 Homo sapiens ankyrin repeat domain 13 family, member D (ANKRD13D), transcript

$25.86 \%$ variant 1 , mRNA

NM_016559.1 Homo sapiens peroxisomal biogenesis factor 5-like (PEX5L), mRNA

$29.07 \%$

homodimeric transcriptional repressors. By regulating these transcriptional factors, diseases are cured following different expression levels of proteins associated with vital physical activities.

In addition to the differentially expressed genes involved in the signaling pathways presented above, there are certain potential marker genes associated with diseases. In conclusion, these genes were classified and concluded according to the effects of curcumin on diseases.

Extensive research has indicated that the antitumor mechanism of curcumin may include the induction of apoptosis of tumor cells, inhibiting various tumor cell growth and proliferation, preventing tumor invasion and metastasis. As a potential anticancer drug, curcumin regulates 21 genes associated with cell cycle and its regulation, differentiation, proliferation and apoptosis. These genes provide further insights into cancer and the likely effects of modifying the encoded targets therapeutically.

Tumor protein p63 (TP63) encodes a member of the p53 family of transcription factors, which functions as a tumor suppressor that is involved in preventing cancer. TP63 may combine with TP73/p73 for initiation of p53/TP53-dependent apoptosis in response to genotoxic insults and the presence of activated oncogenes (15). TP63 may be involved in the notch signaling pathway by possibly inducing Jagged1 (JAG1) and JAG2, and may play a role in the regulation of epithelial morphogenesis (16). The higher expression of TP63 indicates curcumin induced apoptosis of tumor cells and regulated the epithelial tissue and cells by the notch signaling pathway.

Chen and Tan (17) reported that curcumin plays a role in the inhibition of cell growth through inhibiting the c-Jun N-terminal kinase (JNK) pathway and the data of the DNA chip analysis also shows that the mitogen-activated protein kinase binding protein 1 is upregulated by curcumin, which is involved in the JNK signaling pathway to induce apoptosis.

MYC-associated factor $X$ is a member of the basic helix-loop-helix leucine zipper family of transcription factor (18). It is able to form the MYC-MAX complex. Myc is an oncoprotein involved in cell proliferation, differentiation and apoptosis. According to the Kakar and Roy (10) study, the expression of mRNA of myc is lowered only $60 \%$ following incubating with $10 \mathrm{nmol}$ curcumin. In the present study, the expression of the myc gene in the sample with curcumin treatment is only $21.95 \%$ compared to that of the control sample, which proves the same conclusion that curcumin may inhibit the growth of tumor cell by downregulating the myc gene.
There is extensive evidence indicating that the caspase family is essential in cell apoptosis or programmed cell death. The DNA chip analysis reveals that curcumin downregulated the expression of WD repeat domain 35, which functions in the caspase pathway. The same conclusion is reported by Khar et al (19) that the antitumor activity of curcumin functions through the caspase pathway mediated by the induction of apoptosis.

Protein kinase is considered as an extremely important target in the study of antitumor drug. According to Reddy and Aggarwal (20), curcumin may inhibit the activity of a variety of protein kinases involved in tumor cell growth. The DNA chip reveals that numerous curcumin-sensitive genes are associated with protein kinases, such as myotubularin-related protein 4, which functions in phosphorylated on Ser, Thr and Tyr residues and lowered molecular weight phosphatase substrate para-nitrophenylphosphate (21) and GTPase-activating protein binding protein 1 , which may be a phosphorylation-dependent sequence-specific endoribonuclease and involved in the Ras-GTPase pathway by binding specifically to the Ras-GTPase-activating protein (22). The microarray results provide evidence for the Reddy and Aggarwal (20) study that curcumin is an inhibitor of phosphorylase kinase.

The data of the DNA chip showed that curcumin may upregulate heat-shock cognate protein, a member of the heat-shock protein 70 gene family. These proteins play a role in cell proliferation, cell protection, apoptosis and antitumor immune therapy stress response and maintenance of the mitochondria (23). A recent study has proved that heat shock $70 \mathrm{kDa}$ protein 9 (HSPA9) has been shown to interact with fibroblast growth factor 1 (24) and p53 (25), which indicated that HSPA9 and its pathway are promising targets for tumor therapy.

In experiments on tumors, curcumin inhibits the growth of new cancers directly and irreversibly, and it also suppresses the onset of tumors, as well as their growth and metastasis. The present data suggest that curcumin may make important contributions to prevent cancer and decrease cancer mortality through normalizing the expression of tumor-relevant genes.

Curcumin also showed cardioprotective effects by decreasing the level of blood lipid. There are 4 primary genes associated with cardiovascular and cerebrovascular disease that are regulated by curcumin.

A previous study indicated the key contribution of apolipoproteinB (apoB) to atherogenesis as the main inductor of its earlier steps. The apoB receptor is one of the most important elements regarding cholesterol metabolism, which is also a 
curcumin-sensitive element. The apoB receptor is involved in biological processes, such as cholesterol, lipid and steroid metabolism, and lipid transport, while apoB receptor could bind lipoprotein containing apoB48 and apoB100 (26). The present study shows that the level of apoB receptor in L-02 cells treated with curcumin was upregulated 26.07 times compared to the control, which supports considerable evidence for the previous experimental results that curcumin has enough capability to lower the level of low-density lipoprotein (LDL) cholesterol, as well as triglycerides and inhibit LDL oxidation (27) and it would indeed be the novel potential drug in hypercholesterolemia or atherosclerosis therapy. Another study shows that a daily oral administration of the curcumin extract significantly decreases LDL and apoB and increases HDL and apoA of healthy subjects (28).

In conclusion, the present DNA chip data and previous finding of a lowering effect of curcumin on blood lipid and lipoprotein peroxide justifies further study to investigate the molecular mechanism and highly sensitive target of curcumin. Curcumin may be useful as an anti-atherogenic agent.

Inflammation and other immune reactions effect almost all disease progression. Atherosclerosis is an inflammatory disease and the inflammatory cell is an indispensable participant in the neoplastic process, fostering proliferation and survival. According to the data of the DNA chip and research regarding curcumin, curcumin plays an important role in various immune reactions and inflammatory processes.

The data show that curcumin may increase the expression of peptidogly recognition protein 1 (PGLYRP-1), one of the pattern receptors, which is part of the innate immune system and binds to peptidoglycan, a component of bacterial cell walls that has also been found in human atherosclerotic lesions (29). Rohatgi et al (30) found that chronic exposure to bacterial antigens may cause or exacerbate the inflammatory response to lipid deposition within arterial walls, so PGLYRP-1 levels were not only involved in innate immunity, but were also independently associated with atherosclerosis phenotypes. Therefore, it was also suggested that an association may exist between innate immunity and atherosclerosis, and we could draw a conclusion that the upregulation of PGLYRP-1 by curcumin means that curcumin not only prevents bacterial invasion and strong inherent immunity, but also slows down the development of atherosclerosis.

Leptin protein may be involved in the interaction of cytokine receptors, which function as a regulator of immune and inflammatory responses, hematopoiesis, angiogenesis and wound healing (31). As db/db mice have defects of the leptin receptor gene, the leptin protein may be involved in obese type II diabetes with high blood sugar, high cholesterol, insulin resistance and other characteristics. Therefore, the lower expression of leptin protein in the curcumin-treated sample indicates that curcumin may function in reducing cholesterol and blood sugar, reversing insulin resistance.

In the present study, a microarray approach was employed to identify the potential effects of curcumin. The results of the gene chip suggested that curcumin possesses a broad spectrum of biological activities, which includes inhibiting tumor cell growth, antioxidant, anti-inflammatory agent and antithrombotic agent. The biological functions of curcumin are the same as the majority of previous research findings, and additionally, other novel and potential targets spots and pathways have been revealed. All these data construct a genes database regarding curcumin. With further research regarding curcumin, the genes database will be completed and available. From the present study, we can accurately position the target genes and pathway, which supports an overall background of the function of curcumin and make our further research more advisable and appropriate.

The data of the study not only indicated the same conclusion as certain other studies regarding the effects of curcumin, but also revealed novel potential marker genes.

\section{Acknowledgements}

The present study was supported by the Foundation of Zhejiang Provincial Natural Science (grant nos. Y2090891 and LY14H290007), Science Foundation of TCM Administration Bureau of Zhejiang Province (grant no. 2010ZB027) and the Zhejiang Provincial Key Laboratory project (grant no. 2012E10002). Thanks to Mr. Mike Genko Berger (USA) for improving the article language.

\section{References}

1. Sharma RA, Steward WP and Gescher AJ: Pharmacokinetics and pharmacodynamics of curcumin. Adv Exp Med Biol 595: 453-470, 2007.

2. Sa G and Das T: Anti cancer effects of curcumin: Cycle of life and death. Cell Div 3: 14, 2008.

3. Aggarwal BB, Sundaram C, Malani N and Ichikawa H: Curcumin: The Indian solid gold. Adv Exp Med Biol 595: 1-75, 2007.

4. Fan CL, Qian Y, Wo XD, Yan J and Gao LP: Effect of curcumin on the gene expression of low density lipoprotein receptors. Chin J Integr Med 11: 201-204, 2005 (In Chinese).

5. Soni KB and Kuttan R: Effect of oral curcumin administration on serum peroxides and cholesterol levels in human volunteers. Indian J Physiol Pharmacol 36: 273-275, 1992.

6. Peschel D, Koerting R and Nass N: Curcumin induces changes in expression of genes involved in cholesterol homeostasis. J Nutr Biochem 18: 113-119, 2007.

7. King HC and Sinha AA: Gene expression profile analysis by DNA microarrays: Promise and pitfalls. JAMA 286: 2280-2288, 2001.

8. Hong D, Zeng X, Xu W, Ma J, Tong Y and Chen Y: Altered profiles of gene expression in curcumin-treated rats with experimentally induced myocardial infarction. Pharmacol Res 61: 142-148, 2010.

9. Jain SK, Rains J, Croad J, Larson B and Jones K: Curcumin supplementation lowers TNF-alpha, IL-6, IL-8 and MCP-1 secretion in high glucose-treated cultured monocytes and blood levels of TNF-alpha, IL-6, MCP-1, glucose and glycosylated hemoglobin in diabetic rats. Antioxid Redox Signal 11: 241-249, 2009.

10. Kakar SS and Roy D: Curcumin inhibits TPA induced expression of c-fos, c-jun and c-myc proto-oncogenes messenger RNAs in mouse skin. Cancer Lett 87: 85-89, 1994.

11. Neves H, Weerkamp F, Gomes AC, et al: Effects of Delta1 and Jagged1 on early human hematopoiesis: Correlation with expression of notch signaling-related genes in CD34 ${ }^{+}$cells. Stem Cells 24: 1328-1337, 2006.

12. Shukla SD: Platelet-activating factor receptor and signal transduction mechanisms. FASEB J 6: 2296-2301, 1992.

13. Balestrieri ML, Giovane A, Milone L and Servillo L: Endothelial progenitor cells express PAF receptor and respond to PAF via $\mathrm{Ca}(2+)$-dependent signaling. Biochim Biophys Acta 1801: 1123-1132, 2010.

14. Anant JS, Desnoyers L, Machius M, Demeler B, Hansen JC, Westover KD, Deisenhofer J and Seabra MC: Mechanism of Rab geranylgeranylation: Formation of the catalytic ternary complex. Biochemistry 37: 12559-12568, 1998.

15. Yang A, Kaghad M, Wang Y, Gillett E, Fleming MD, Dötsch V, Andrews NC, Caput D and McKeon F: p63, a p53 homolog at 3q27-29, encodes multiple products with transactivating, death-inducing, and dominant-negative activities. Mol Cell 2: 305-316, 1998. 
16. Hau PM, Yip YL, Huen MS and Tsao SW: Loss of $\triangle \mathrm{Np} 63 a$ promotes mitotic exit in epithelial cells. FEBS Lett 585: 2720-2726, 2011.

17. Chen YR and Tan TH: Inhibition of the c-Jun N-terminal kinase (JNK) signaling pathway by curcumin. Oncogene 17: 173-178, 1998.

18. Cotterman R, Jin VX, Krig SR, Lemen JM, Wey A, Farnham PJ and Knoepfler PS: N-Myc regulates a widespread euchromatic program in the human genome partially independent of its role as a classical transcription factor. Cancer Res 68: 9654-9662, 2008

19. Khar A, Ali AM, Pardhasaradhi BV, et al: Antitumor activity of curcumin is mediated through the induction of apoptosis in AK-5 tumor cells. FEBS Lett 445: 165-168, 1999.

20. Reddy S and Aggarwal BB: Curcumin is a non-competitive and selective inhibitor of phosphorylase kinase. FEBS Lett 341: 19-22, 1994.

21. Zhao R, Qi Y, Chen J and Zhao ZJ: FYVE-DSP2, a FYVE domain-containing dual specificity protein phosphatase that dephosphorylates phosphotidylinositol 3-phosphate. Exp Cell Res 265: 329-338, 2001.

22. Vognsen T, Möller IR and Kristensen O: Purification, crystallization and preliminary X-ray diffraction of the G3BP1 NTF2-like domain. Acta Crystallogr Sect F Struct Biol Cryst Commun 67: 48-50, 2011.

23. Bhattacharyya $T$, Karnezis AN, Murphy SP, Hoang $T$, Freeman BC, Phillips B and Morimoto RI. Cloning and subcellular localization of human mitochondrial hsp70. J Biol Chem 270: 1705-1710, 1995.

24. Mizukoshi, E, Suzuki M, Loupatov A, Uruno T, Hayashi H, Misono T, Kaul SC, Wadhwa R and Imamura T: Fibroblast growth factor-1 interacts with the glucose-regulated protein GRP75/mortalin. Biochem J 343: 461-466, 1999.
25. Wadhwa R, Yaguchi T, Hasan MK, Mitsui Y, Reddel RR and Kaul SC: Hsp70 family member, mot-2/mthsp70/GRP75, binds to the cytoplasmic sequestration domain of the p53 protein. Exp Cell Res 274: 246-253, 2002.

26. Sberna AL, Assem M, Xiao R, Ayers S, Gautier T, Guiu B, Deckert V, Chevriaux A, Grober J, Le Guern N, et al: Constitutive androstane receptor activation decreases plasma apolipoprotein B-containing lipoproteins and atherosclerosis in low-density lipoprotein receptor-deficient mice. Arterioscler Thromb Vasc Biol 31: 2232-2239, 2011.

27. Olszanecki R, Jawień J, Gajda M, Mateuszuk L, Gebska A, Korabiowska M, Chłopicki S and Korbut R: Effect of curcumin on atherosclerosis in apoE/LDLR-double knockout mice. J Physiol Pharmacol 56: 627-635, 2005.

28. Ramírez-Boscá A, Soler A, Carrión MA, Díaz-Alperi J, Bernd A, Quintanilla C, Quintanilla Almagro E and Miquel J: An hydroalcoholic extract of curcuma longa lowers the apo B/apo A ratio. Implications for atherogenesis prevention. Mech Ageing Dev 119: 41-47, 2000.

29. Liu C, Xu Z, Gupta D and Dziarski R: Peptidoglycan recognition proteins: A novel family of four human innate immunity pattern recognition molecules. J Biol Chem 276: 34686-34694, 2001.

30. Rohatgi A, Ayers CR, Khera A, McGuire DK, Das SR, Matulevicius S, Timaran CH, Rosero EB and de Lemos JA: The association between peptidoglycan recognition protein-1 and coronary and peripheral atherosclerosis: Observations from the Dallas Heart Study. Atherosclerosis 203: 569-575, 2009.

31. Sierra-Honigmann MR, Nath AK, Murakami C, García-Cardeña G, Papapetropoulos A, Sessa WC, Madge LA, Schechner JS, Schwabb MB, Polverini PJ, et al: Biological action of leptin as an angiogenic factor. Science 281: 1683-1686, 1998. 